\title{
Biventricular pacing in heart failure: right is not wrong!
}

\author{
Prem Soman
}

Published online: 3 April 2014

(C) Springer-Verlag Berlin Heidelberg 2014

Cardiac resynchronization therapy (CRT) improves left ventricular (LV) function, symptoms, and prognosis in selected patients with heart failure [1-3]. Those with left bundle branch block (LBBB) and the widest QRS complexes seem to have the highest rate of response [4]. However, a significant percentage of patients with guideline-approved criteria remain "nonresponders." Improving the CRT response rate using imaging techniques has remained an elusive goal. This is a worthy area of research since a suboptimal response rate affects the cost-effectiveness of CRT unfavorably and subjects patients to the risks of the procedure without its benefits.

Our current knowledge base of CRT mechanisms indicates that response depends on a complex interplay among many known and potentially unknown factors [5]. Despite a substantial body of literature attesting to the benefits of CRT in selected patients with heart failure, surprisingly little data exist pertaining to the mechanisms of benefit. One presumes logically that CRT produces its salutary effects by improving LV mechanical synchrony, but the large multicenter studies that established the field did not explore mechanisms.

Several small, single-center studies have explored CRT mechanisms. In a study of 100 patients chosen for conventional CRT indications and the presence of LV dyssynchrony defined as opposing wall delay of $>65 \mathrm{~ms}$ on tissue Doppler echocardiography, Bleeker and colleagues from Leiden reported that a significant improvement in LV synchrony after CRT was mandatory for chronic remodeling effects to occur [6]. They also found that when resynchronization occurred it was evident immediately, and there was little if any "recruitment" on follow-up. A pilot study from our group suggested that the acute response to CRT can be heterogeneous in patients

P. Soman $(\bowtie)$

Division of Cardiology, University of Pittsburgh, A-429, Scaife Hall, 200 Lothrop Street, Pittsburgh, PA 15238, USA

e-mail: somanp@upmc.edu undergoing CRT for guideline-based indications, with approximately one third of patients having worse LV synchrony after CRT [7]. Patients who had significant LV dyssynchrony, noncritical extent and location of LV scar, and a strategic LV lead position in viable, late-activated myocardium had the highest chance of improved synchrony after CRT. Importantly, patients in whom LV synchrony deteriorated after CRT had a worse composite outcome compared to patients who improved or remained unchanged. A positive association between each of the above component requirements for successful acute resynchronization and a favorable long-term outcome has been demonstrated in other small and mostly retrospective studies [8-10]. In the longer term, the acute salutary effect of CRT on LV dyssynchrony appears to be associated with chronic LV reverse remodeling and improved prognosis [11]. Thus, the mere presence of LV dyssynchrony at baseline may not translate into a benefit of CRT, but rather a constellation of conditions should be met to make the dyssynchrony remediable.

In this issue of the European Journal of Nuclear Medicine and Molecular Imaging, Hung and colleagues report their exploration of an interesting and clinically relevant concept [12]. They argue that while the measurement and characterization of LV dyssynchrony are usually performed in the native state [except in patients with a right ventricular (RV) pacemaker], the net effect of CRT on LV synchrony must surely be the result of interplay between RV and LV pacing. This is certainly a logical construct. The investigators use a single photon emission computed tomography (SPECT)-based approach which was pioneered by the senior author of this manuscript. The excellent repeatability of these automated measurements in combination with the "single-injection protocol" developed in our laboratory at the University of Pittsburgh makes the approach taken by these investigators uniquely suited to measure acute changes in LV synchrony after CRT with minimal radiation to the patient. Thus, 46 
patients in sinus rhythm who had previously undergone CRT for standard indications were studied in the native state and during RV pacing. Both images were sequentially acquired 30 min apart after one single injection of ${ }^{99 \mathrm{~m}} \mathrm{Tc}$-sestamibi. The site of optimal LV lead position was defined on an eightsegment LV model as the segment with the latest activation (largest mean phase) that was viable ( $<50 \%$ scar). The main finding of the study was that in the majority of patients ( $84 \%)$ the segment with the most delayed activation was the same during sinus rhythm and RV pacing. In six patients, the segment of latest activation was shifted towards the base in the same radial direction (anterior, anterolateral, posterior, or posterolateral). In only one patient with a large scar was the segment of latest activation completely different between sinus rhythm and RV pacing. As expected, there was greater LV dyssynchrony during RV pacing compared to sinus rhythm.

Are these findings tenable with the physiology of LBBB and RV pacing? As illustrated in Fig. 1 by the authors, it is conceivable that in patients with LBBB the site of origin of LV activation is not dissimilar during sinus rhythm and RV apical pacing. Furthermore, animal studies have shown that the "sweet spot" of optimal LV pacing might actually be quite a large area of the lateral wall [13]. Given that our ability to deliver CRT by multisite pacing is currently limited to biventricular pacing of the RV apex or septum and a limited number of LV lateral wall positions dictated by coronary venous anatomy, the finding of a consistent site of latest LV activation during native LBBB rhythms and RV pacing suggests a role for serendipity in addition to physiology in explaining the effects of CRT. All that contemporary CRT probably does is to correct a septal to lateral activation delay, and thus it produces the highest response rates in patients with the widest QRS complexes and LBBB. In these patients, imaging may offer little advantage over clinical criteria for the selection of patients for CRT. In fact, the adaptive CRT trial results suggest that CRT with an LV lead alone might produce even better results than biventricular pacing in patients with standard indications [14].

It must be remembered that the study by Hung and colleagues is a small one with a relatively homogeneous population. Elegant studies have demonstrated that left-sided septal activation and its subsequent spread to the LV free wall may in fact vary among patients with LV systolic dysfunction and LBBB $[15,16]$. Rodriguez and colleagues showed that LV activation in patients with LBBB could occur either by slow conduction from branches of the left bundle or by right-to-left transseptal conduction. Additional conduction delay beyond the bundle branch occurred within the LV myocardium in scarred or myopathy ventricles. However, in all 12 patients with LBBB in that study, the latest LV activation occurred in the posterobasal or posterolateral wall [15]. Thus, larger studies may in fact confirm the generalizability of the findings of
Hung and colleagues. In the meantime, the authors should be commended for using a creative protocol to explore a logical question.

\section{References}

1. Abraham WT, Fisher WG, Smith AL, Delurgio DB, Leon AR, Loh $\mathrm{E}$, et al. Cardiac resynchronization in chronic heart failure. N Engl J Med 2002;346:1845-53.

2. Cleland JGF, Daubert JC, Erdmann E, Freemantle N, Gras D, Kappenberg L, et al. The effect of cardiac resynchronization on morbidity and mortality in heart failure. N Engl J Med 2005;352: 1539-49.

3. Moss AJ, Hall WJ, Cannom DS, Klein H, Brown MW, Daubert JP, et al. Cardiac-resynchronization therapy for the prevention of heartfailure events. N Engl J Med 2009;361:1329-38.

4. Zareba W, Klein H, Cygankiewicz I, Hall WJ, McNitt S, Brown M, et al. Effectiveness of cardiac resynchronization therapy by QRS morphology in the multicenter automatic defibrillator implantation trial-cardiac resynchronization therapy (MADIT-CRT). Circulation 2011;123:1061-72.

5. Soman P, Schwartzman D. Dyssynchronous! Remediable? J Nucl Cardiol 2012;19:11-2.

6. Bleeker GB, Mollema SA, Holman ER, Van de Veire N, Ypenburg C, Boersma $\mathrm{E}$, et al. Left ventricular resynchronization is mandatory for response to cardiac resynchronization therapy: analysis in patients with echocardiographic evidence of left ventricular dyssynchrony at baseline. Circulation 2007;116:1440-8.

7. Friehling M, Chen J, Saba S, Bazaz R, Schwartzman D, Adelstein $\mathrm{EC}$, et al. A prospective pilot study to evaluate the relationship between acute change in left ventricular synchrony after cardiac resynchronization therapy and patient outcome using a singleinjection gated SPECT protocol. Circ Cardiovasc Imaging 2011;4: 532-9.

8. Auger D, Bleeker GB, Bertini M, Ewe SH, van Bommel RJ, Witkowski TG, et al. Effect of cardiac resynchronization therapy in patients without left intraventricular dyssynchrony. Eur Heart J 2012;33:913-20.

9. Adelstein EC, Tanaka H, Soman P, Miske G, Haberman SC, Saba SF, et al. Impact of scar burden by single-photon emission computed tomography myocardial perfusion imaging on patient outcomes following cardiac resynchronization therapy. Eur Heart J 2011;32:93-103.

10. Ypenburg C, van Bommel RJ, Delgado V, Mollema SA, Bleeker GB, Boersma E, et al. Optimal left ventricular lead position predicts reverse remodeling and survival after cardiac resynchronization therapy. J Am Coll Cardiol 2008;52:1402-9.

11. Yu CM, Bleeker GB, Fung JW, Schalij MJ, Zhang Q, van der Wall $\mathrm{EE}$, et al. Left ventricular reverse remodeling but not clinical improvement predicts long-term survival after cardiac resynchronization therapy. Circulation 2005;112:1580-6.

12. Hung G-U, Huang J-L, Lin W-Y, Tsai S-C, Wang K-Y, Chen S-A, et al. Impact of right-ventricular apical pacing on the optimal leftventricular lead positions measured by phase analysis of SPECT myocardial perfusion imaging. Eur $\mathrm{J}$ Nucl Med Mol Imaging 2014;41. doi:10.1007/s00259-014-2693-y

13. Helm RH, Byrne M, Helm PA, Daya SK, Osman NF, Tunin R, et al. Three-dimensional mapping of optimal left ventricular pacing site for cardiac resynchronization. Circulation 2007;115:953-61.

14. Birnie D, Lemke B, Aonuma K, Krum H, Lee KL, Gasparini M, et al. Clinical outcomes with synchronized left ventricular pacing: analysis of the adaptive CRT trial. Heart Rhythm 2013;10:1368-74. 
15. Rodriguez LM, Timmermans C, Nabar A, Beatty G, Wellens HJ. Variable patterns of septal activation in patients with left bundle branch block and heart failure. J Cardiovasc Electrophysiol 2003;14:135-41.
16. Vassallo JA, Cassidy DM, Miller JM, Buxton AE, Marchlinski FE, Josephson ME. Left ventricular endocardial activation during right ventricular pacing: effect of underlying heart disease. J Am Coll Cardiol 1986;7:1228-33. 